\title{
Caracterización del proceso de conurbación fronteriza: el caso de Leticia (Colombia) y Tabatinga (Brasil)
}

\author{
Characterization of the process of border suburbs: \\ case of Leticia (Colombia) and Tabatinga (Brasil)
}

Pablo Enrique Castillo Gutiérrez *

Citar este artículo como: Castillo Gutiérrez, P. E. (2018). Caracterización del proceso de conurbación fronteriza: el caso de Leticia (Colombia) y Tabatinga (Brasil) Revista Nodo, 12(24), 46-59.

\section{Resumen}

El presente artículo pretende resumir el trabajo de investigación adelantado con el objetivo de definir, caracterizar y apropiar los elementos conceptuales que permitan describir el fenómeno de ocupación territorial desde la óptica de la sostenibilidad, el urbanismo, la cooperación fronteriza transnacional, la habitabilidad y la construcción social del hábitat territorial sostenible; en el marco de la multiculturalidad, lo patrimonial y la concepción del buen vivir de la franja de territorio limítrofe binacional de Leticia en Colombia y Tabatinga en el Brasil, ubicado en las márgenes del río Amazonas.

Palabras clave: Resiliencia urbana, Hábitat saludable, Construcción social, Conurbación, Cooperación transfronteriza

\section{Abstract}

This article is intended to summarise the research process so-far developed in order to: define, characterise and appropriate concep- tual elements, which allows one to describe the phenomenon of territorial occupation, from the perspective of sustainability, the Urbanism, the trans-border co-operation, habitability and the Social construction of the Habitat Territorial sustainable; within the framework of multiculturalism, the heritage and the conception of the good life; and the strip of binational border territory, Leticia in Colombia and Tabatinga in the Brazil, located on the Bank of the Amazon River.

Keywords: Urban resilience, Healthy habitat, Social construction, Conurbation, Cross-Border Cooperation

\section{Introducción}

Esta investigación parte de un estudio previo que ha venido adelantando el suscrito en la ciudad de Leticia sobre la caracterización del Hábitat en la zona de borde entre el casco urbano de Leticia y el río Amazonas, así como del conocimiento del territorio dada la experiencia de trabajo con entidades de orden nacional en temas de vivienda de

Fecha de recibo: 25 de julio de 2018 • Fecha de aceptación: 1 de octubre de 2018

\footnotetext{
* $\quad$ Arquitecto Investigador en Asuntos Amazónicos y docente de la Universidad la Gran Colombia. Correo electrónico: pecg17@gmail.com; pablo.castillo@ugc.edu.co
} 
interés social y en calidad de consultor, contratado por la Alcaldía de Leticia para adelantar varios estudios de saneamiento básico, así como indicadores cualitativos y cuantitativos del hábitat en la ciudad del Leticia.

Este estudio pretende generar una mayor comprensión de la caracterización del hábitat en la frontera colombo brasilera, particularmente entre las ciudades de Leticia y Tabatinga. Esto a partir de las potencialidades propias de cada territorio nacional que al producir alianzas de planificación y gestión territorial desde el concepto de hábitat territorial sostenible, permite generar una mayor calidad de vida para los pobladores de dichos lugares.

La estructura de este trabajo comprende básicamente cuatro (4) directrices de investigación, a saber:

- Hábitat saludable en los usos urbanos tanto en el domicilio como en el peri-domicilio, bajo la visión de lo ambiental, los riesgos eco sistémicos y los vectores generadores de afecciones a los seres vivos, dando a su vez las recomendaciones para un control efectivo desde el buen manejo del entorno habitable y de sus indicadores mínimos de calidad, presentes entre otros en los estudios hechos por la Organización Panamericana de la Salud y la Organización Mundial de la Salud (OMS) que presentan una estrategia de vivienda saludable hacia la mejora de los asentamientos y del subsistema físico biótico en general, para mejorar espacios tales como la casa o habitáculo, considerados como los refugios físicos donde habitan las personas; el hogar o grupo de personas que viven bajo el mismo techo y su entorno o el ambiente exterior que rodea la casa y la comunidad o grupo de personas que constituyen el vecindario.

- Análisis socio espacial de las formas de articular y definir la transnacionalidad territorial en la franja de frontera a lo largo de su cartografía espacial y la manera de ocupar el territorio.

- Parámetros de los dos (2) países para la organización y geopolítica del espacio geográfico y cómo esto permea la población y el espacio socio productivo.

- Buen vivir: se relaciona con la forma como estos pobladores, muchos de ellos de condición indígena, quienes en su organización en la construcción de sociedad sobre este territorio, han definido los llamados "Planes de Vida" los cuales se soportan legalmente en el artículo 330 de la Constitución de Colombia, dentro de las funciones de los gobiernos indígenas. Esto se enfoca igualmente en sus propias políticas, planes y programas de desarrollo económico. Para esto me permito citar a Azcaita (2008):

(...) Plan de vida, algo nuevo en palabras, pero eterno en vivencias de todos los días, se desarrolla en cada planta, en los animales y seres humanos (...) Los planes integrales de vida promueven una visión de la vida diferente del concepto de desarrollo occidental al incluir especialmente el fortalecimiento organizativo, cultural, político y espiritual, como parte de una filosofía del buen vivir con la familia, la comunidad y la naturaleza. (pp. 9-16).

De acuerdo a lo anterior se plantea la pregunta: ¿qué elementos de integración transfronteriza del hábitat territorial sostenible, se pueden identificar, para definir y calificar la franja territorial de borde, que permita caracterizar el espacio como elemento fundamental de construcción social y física del territorio a partir de su caracterización de hábitat desde o saludable y desde su particularidad? Un ejemplo de ello corresponde a la apropiación de dicho territorio a partir de una cultura transnacional y trifronteriza (Colombia, Brasil, Perú) que comprende procesos de movilidad e intercambio cultural, productivo $o$ básicamente el de "estar allí" como construcción social de ese territorio periférico a través de sus modos de vida y modos de habitarlo y, a la vez, de transformarlo permanentemente en su estructura ecológica y en su interfaz biótica. Dándose entonces un fenómeno denominado "Ciudades Gemelas", como posible catalizador de cooperación fronteriza. 


\section{Contexto}

$\mathrm{Al}$ sur de Colombia, en el extremo meridional del trapecio amazónico, se localiza la ciudad de Leticia, capital del departamento del Amazonas, que limita por el costado sur con el río Amazonas, el cual nace en los altos paramos del Perú y culmina su recorrido en la población brasileira de Belén do Pará. En Leticia recibe el nombre de río Solimues entrando al territorio brasileiro, donde empalma con el río Putumayo, generando una interconectividad fluvial, para transporte de mercancía y otros bienes entre las poblaciones aledañas a sus afluentes en ambos países.

La caracterización de esta zona se estructura desde la metodología del "Estudio de Zonas de Frontera" realizada por el gobierno de Brasil, la cual define indicadores de las condiciones materiales de vida, accesibilidad y disponibilidad de servicios de salud, complementado con datos de poblamiento, migración e información recogida sobre el terreno en cinco puntos distintos de la frontera en el período comprendido entre el 2001 al 2006 y que suministra información para las ciudades de Tabatinga (Brasil) y Leticia (Colombia); determinándose de esa manera las franjas de frontera de Brasil: arcos y subregiones.

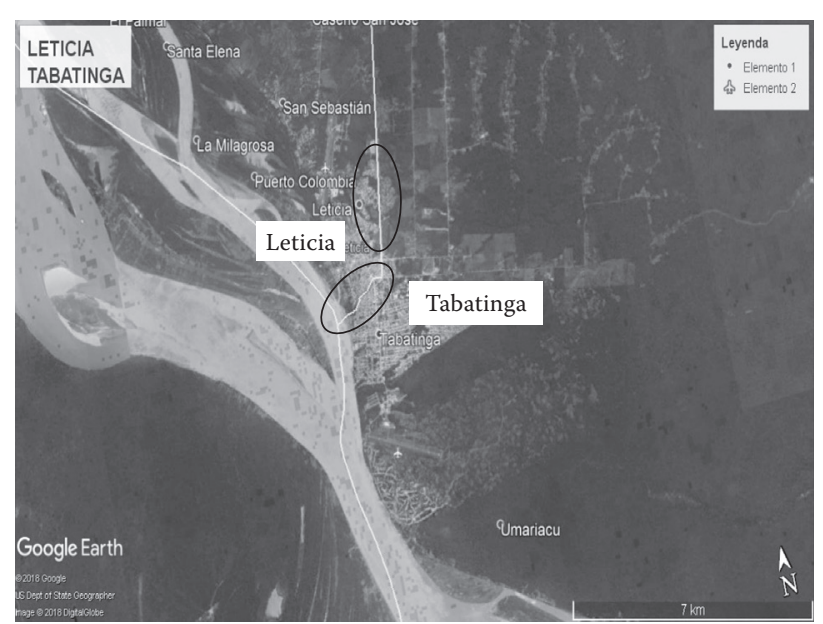

Figura 1. Ciudades transfronterizas. Sectores conurbados y micro zonas de construcción territorial. Fuente: Adaptación propia a partir de Google maps

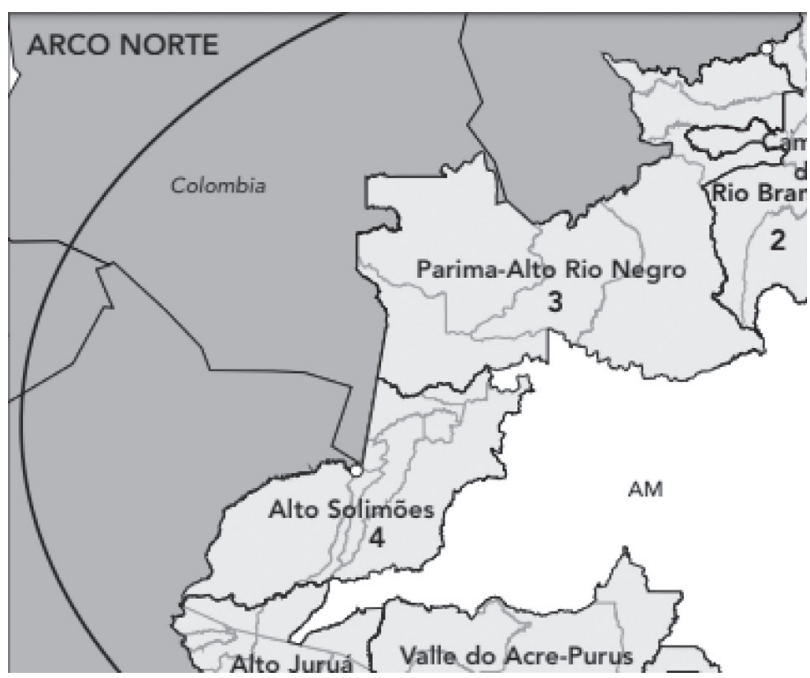

Figura 2. Franja de Frontera (Brasil): aspectos del poblamiento de las subregiones, 2000. Fuente: Ministerio de Integración Regional; Grupo Retis/Universidad Federal de Río de Janeiro, 2005

\section{Antecedentes}

Durante los últimos 50 años, América Latina ha sido testigo de múltiples procesos de ocupación territorial en las fronteras, de forma particular y adaptada a las condiciones de cada localidad. Para el caso objeto de este estudio, me baso en un criterio de análisis territorial integracionista desde el concepto de Territorio Transnacional y en la identificación de factores de habitabilidad de esta zona urbana, caracterizando entre otros aspectos las dinámicas de construcción social del territorio y articulando esto al estudio de roles de sus pobladores, así como a elementos de integración urbanística de las dos ciudades fronterizas para esbozar un planteamiento integral de mejor calidad de vida.

Se trata entonces de abordar el fenómeno de urbanización de la Amazonía a partir del concepto de Anillo de Poblamiento, con pocos estudios que lo denoten, a excepción de lo producido juiciosamente por el Instituto Amazónico de Investigaciones Científicas Sinchi. (Riaño \& Salazar, 2009).

En este sentido se parte básicamente del conocimiento de la historia sucinta de estos asentamientos y teniendo en cuenta que su reciente 
evolución no solo proviene de los eventos de ocupación fronteriza dados a manera de conflicto limítrofe con el Perú en el caso de Colombia, sino más bien de externalidades actuales, representadas por los agentes consulares de fronteras, los misioneros y los eventos de ocupación territorial por migrantes con diversos intereses, entre éstos, los derivados de procesos de desplazamiento o de formas de ocupación territorial ocasionados por la carencia de vivienda, baja oferta de ésta en las localidades o incluso conveniencias que permiten vivir en la precariedad mientras se dan condiciones de lucro mediante la renta de bienes inmuebles en zonas más acomodadas de las ciudades. Así mismo es factor a considerar el poco control normativo y estatal a la ocupación espontánea y no planificada y a preceptos individualistas y no de ordenamiento colectivo.

Se sugiere proyectar líneas estratégicas constructivas a partir del reconocimiento multinivel y multirealista de la historia de los moradores y generadores del fenómeno de frontera y de conurbación transnacional. Sin dejar de lado la gran incidencia del componente étnico. Destacándose entonces el hecho de reconocer que la pertinencia fronteriza se caracterizó en el caso de Colombia por intereses propios de las misiones religiosas, pasando a la presencia gubernamental y permeada recientemente por diversos fenómenos sociales; mientras que, en el caso del Brasil, históricamente se ha dado un proceso de táctica militar, construcción de asentamientos de defensa y, recientemente la presencia militar y político-administrativa dentro de un concepto bastante claro de planificación territorial de las fronteras (Zárate, 2008).

Estos asentamientos localizados en el extremo sur del Trapecio Amazónico presentan antecedentes de ocupación territorial relativamente recientes (siglo XVI), por parte de algunas etnias, especialmente en la región ubicada entre el bajo río Napo (Perú) y la región de São Paulo de Olivença (Brasil), produciéndose posteriormente un fuerte proceso de evangelización portuguesa que hacia
1766 finalizó con la creación del fuerte militar de São Francisco Xavier de Tabatinga materializando de esta forma sus conquistas.

Posteriormente los peruanos bajo la tutela del capitán Benigno Bustamante fundaron hacia el año de 1867 el puerto quinero de Leticia, funcionando en calidad de Puesto Militar de San Antonio, colindante con el ya mencionado fuerte militar de São Francisco Xavier de Tabatinga.

Es de destacar el momento histórico entre 1850 y 1930 en el que se presentó una especial demanda de productos silvestres tropicales, entre ellos las gomas de látex o caucho, tales como el Hevea brasilensis, propiciándose un ambiente de conflictos transnacionales entre empresarios peruanos que, empoderadas por la empresa Casa Arana, asesinaron a los empresarios colombianos y explotaron inhumanamente a los indígenas sin que se pronunciara sobre ello el gobierno de Colombia de ese entonces.

Ya en el año de 1922 se delimitó la frontera con el Perú a través tratado Lozano-Salomón asumiendo el trapecio amazónico como jurisdicción colombiana siendo entonces declarado el territorio en 1928 como comisaría especial del Amazonas y su capital la población de Leticia; sin embargo, el señor Julio César Arana y otros políticos de Iquitos se tomaron Leticia en 1.932, generando el largo conflicto peruano - colombiano que terminó en 1.933 con el Protocolo de Río de Janeiro a favor de Colombia.

En dicho contexto es de destacar que inicialmente se dio la creación del poblado El Marco en el costado Brasileño de la Quebrada San Antonio, a través del Tratado García Ortíz-Mangabeira en 1928, dando origen a surgimiento del marco en 1932, donde se asentaron pobladores que venían del Yavarí, la mayoría caucheros en crisis.

Es de destacar cómo los flujos recientes consolidados de los dos poblados, entre 1950 y 1960, determinaron gran parte la forma de ocupación de la franja de frontera donde a pesar de haberse dado la construcción de la avenida internacional 
en Leticia que conectó a la "Avenida a Amizade" en Tabatinga como único punto de ingreso formal binacional, se dio un fenómeno interesante de ocupación en el borde fronterizo de un sendero para la circulación entre los dos países llamado "caminito de Dídimo", que continuó un buen tiempo funcionando como tal con un elemento de relieve muy particular en su cruce con la Quebrada San Antonio. Esto provocó prácticas específicas de construcción social de los lugares aledaños al mismo. Posteriormente, se selló tal paso con el predio de la Central Termoeléctrica de Leticia y por la política binacional de unificar un paso único por el costado Oriental (Picór Acuña, 2010, pp. 113-114). En esas mismas décadas se generaron procesos de explotación y rutas de salida de pieles, animales y madera y finalizando los años 70 así como durante la década de los años 80 , dichas rutas se aprovecharon para el tráfico de drogas; sin embargo en los años 90 se disolvió el cartel de la droga en Leticia y se confiscaron los bienes producto de dineros ilegales. A partir de los años 90 y en lo que va del siglo XXI se observa un proceso de estabilización económica en actividades tales como la pesca y la venta de servicios turísticos y gastronómicos (Riaño Umbarila, 2009).

En las décadas de los años sesenta 60 y 70, se generaron procesos de explotación y rutas de

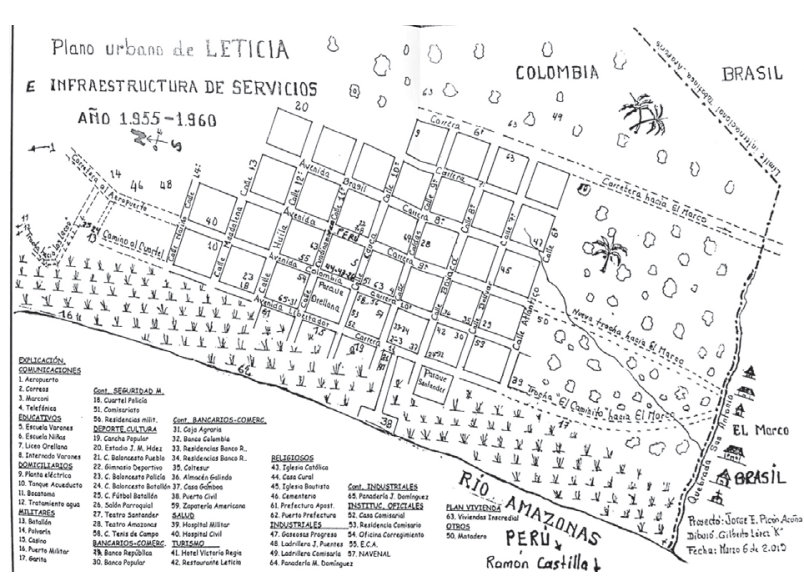

Figura 3. Plano Urbano de Leticia 1955 y 1962 con servicios públicos. Fuente: Picón Acuña, 2010 salida de pieles, animales y madera y finalizando los años 70 así como durante la década de los años 80, dichas rutas se aprovecharon para el tráfico de drogas. Más ya en los años 90 se disolvió el cartel de la droga en Leticia y se confiscaron los bienes producto de dineros ilegales.

Posteriormente, en los años 90 y del año 2000 a la fecha, se observa un proceso de estabilización económica en actividades tales como la pesca y la venta de servicios turísticos y gastronómicos. (Riaño Umbarila, 2009, p. 233).

\section{Apuntes sobre la metodología}

Se utilizaron estrategias metodológicas tales como revisión y consulta de fuentes de información primaria y secundaria que apoyadas en la observación directa del territorio y a partir del conocimiento previo del contexto y la verificación actual in situ, permitió la consolidación de un corpus de datos significativo.

En ese sentido también se consultaron fuentes de referencia entre ellas artículos especializados tanto colombianos como brasileros y documentos legales de soporte normativo en la planificación territorial.

Y, por último, se determinaron zonas características o polígonos en el territorio de estudio de acuerdo al comportamiento y caracterización de cada cual, tales como la desembocadura de la quebrada San Antonio en el río Amazonas; el borde fronterizo entre el barrio Castañal en Leticia y los hábitats sobre la Rua Ml; Rondon en Tabatinga y finalmente, la franja fronteriza entre el Iane y Ciudad Nueva en Leticia, con los terrenos brasileros que están siendo descapotados y ocupados en forma acelerada.

\section{Resultados y hallazgos: la conurbación transfronteriza}

Se determina una perspectiva multiescalar que necesariamente aplica a la concreción de los 
factores constructores de dicha transnacionalidad, teniendo en cuenta la incidencia desde lo macro a lo micro y a partir de los elementos definitorios de la territorialidad por parte de cada país, especialmente en lo que respecta a los siguientes temas:

- Conceptos de seguridad ambiental global y de desarrollo sustentable "...los cuales son argumentos centrales en la construcción del orden ambiental internacional, amalgama que combina el mantenimiento de las condiciones de reproducción de vida humana en la tierra con regulación sustentable de los recursos naturales..." (Ribeiro, 2001, p. 109). En tanto que “(...) la configuración pluridimensional de la seguridad avanza y amplía el debate, permitiendo analizar los riesgos de inseguridad crecientes a principios de este siglo. Esto se puede reflejar sobre la seguridad militar, seguridad económica, seguridad ambiental y seguridad societaria. Esa visión considera que la seguridad militar continúa siendo central, aunque no es la única a ser garantizada..." (Teixeira da Silva, 2009, p. 20)

- Conceptos sobre instrumentos de gestión territorial que afectan esta frontera, como es el caso del OTCA (Tratado de Cooperación Amazónica), en el que se parte de propósitos proactivos dados por las Repúblicas de Bolivia, Brasil, Colombia, Ecuador, Guyana, Perú, Surinam y Venezuela; dentro de un proceso de cooperación que redundará en beneficio de sus respectivos países y de la Amazonia en su conjunto, de los cuales se destacan como puntos aplicables a la franja de estudio, los que se evidencian en la siguiente tabla:

La caracterización de esta zona se estructura desde la óptica del gobierno brasileño que determina que el costado del Brasil sobre la franja Norte

Tabla 1. Cuadro aplicativo de preceptos Tratado de Cooperación Amazónica en la zona de estudio

\section{Mención en el tratado de cooperación amazónica - Declaración de la Amazonía}

"Las Partes Contratantes procurarán empeñar esfuerzos con miras a la utilización racional de los recursos hídricos".

“...Promover la investigación científica y el intercambio de informaciones y de personal técnico entre las entidades competentes de los respectivos países a fin de ampliar los conocimientos sobre los recursos de la flora y de la fauna de sus territorios amazónicos y prevenir y controlar las enfermedades en dichos territorios..."

“...EXPRESAMOS nuestro respaldo a las Comisiones Especiales del Medio Ambiente y de Asuntos Indígenas, recientemente creadas, destinadas a impulsar el desarrollo, conservar los recursos naturales, el medio ambiente y las respectivas poblaciones amazónicas y
Aplicabilidad a la franja de frontera brasil - Colombia Tabatinga - Leticia, propuesta por el autor

Generar acuerdos fronterizos en cuidado y preservación de la quebrada San Antonio, como contexto natural eco sistémico común a ambos países. Esto a través de entidades locales como la Alcaldía mayor de Leticia, junto con la Prefeitura Municipal de Tabatinga. Sin embargo se debe verificar esta Gestión ya que en el sitio web de esta última figuran activos solo los Convenios Federales y los Estaduales.

Con fines de desarrollar estudios e investigaciones como la presente, con miras a implementar a través de entidades y/o gestores de ambos países tales como la Academia, las organizaciones no gubernamentales, entre otras, para normalizar y normatizar el territorio, en calidad de instrumentos de Gestión Territorial y control a la degradación del hábitat.

Implementación de comisiones transnacionales que deriven en grupos de construcción y cuidado social del territorio, con productividad social y organización comunitaria programática, focalizando las acciones en los grupos indígenas previamente censados, registrados y comunicados, 
Mención en el tratado de cooperación amazónica Declaración de la Amazonía

reiteramos el pleno respeto al derecho que asiste a las poblaciones indígenas de los territorios amazónicos a que se adopten todas las medidas conducentes para mantener y preservar la integridad de estos grupos humanos, sus culturas y su hábitat ecológico, en el ejercicio del derecho inherente a la soberanía de cada Estado..."

“... RECONOCEMOS que la defensa de nuestro medio ambiente requiere del estudio de medidas, bilaterales y regionales, para prevenir accidentes contaminantes y atender sus consecuencias..."

Es fundamental combatir la sequía y conservar las cuencas hidrográficas mediante el aprovechamiento integrado de los recursos del suelo e hídricos y el mantenimiento de la cobertura vegetal.

El conocimiento cabal de la realidad de cada uno de los territorios amazónicos y de las diferencias y características de ellos para el uso racional de sus recursos naturales para el aprovechamiento agroindustrial, pecuario e hidroeléctrico, se verá facilitado en la medida en que los países amazónicos logren niveles de avances científicos y tecnológicos que afirmen su solvencia y autonomía. Con este objetivo, y para una mejor percepción de la realidad amazónica, se estimulará a las universidades de los países miembros a establecer centros o asignaturas de estudios amazónicos, como ya existen en algunas, y a promover la intensificación de contactos entre dichos centros de estudios, profesores, investigadores, artistas, intercambio de becarios y divulgación de publicaciones sobre temas amazónicos.

Fuente: Tratado Cooperación Amazónica y elaboración propia

del Alto Solimues acusa condiciones precarias en términos de salud, vivienda e infraestructura junto al acceso a servicios básicos domiciliares, al igual que al control ambiental ecosistémico del lugar.

\section{Aplicabilidad a la franja de frontera brasil - Colombia Tabatinga - Leticia, propuesta por el autor}

entre ellos los Yaguas y Ticunas, en un trabajo mancomunado con los actuales moradores de la franja de frontera urbana transnacional; diferenciando en todo caso el cuidado y preservación ecológica y eco sistémica de la soberanía de cada país en particular.

Generación de planes de gestión ambiental, apoyados por instituciones locales (Corpoamazonía, Secretaria Municipal de Medio Ambiente), de los dos (2) países y por los estamentos de nivel central de cada uno de ellos y sus respectivos Ministerios del medio ambiente.

Generación de mecanismos de control a la disminución de la cobertura vegetal aledaña a las cuencas hídricas de diversas escalas, con énfasis en la Quebrada San Antonio y su desembocadura en el río Amazonas, con acompañamiento a veedores y guarda cuencas que moren en los asentamientos ya indicados, tanto en Colombia (comunidad Indígena Yagua, barrio la Unión) como en Brasil (barrios Guadalupe, Sao Francisco, el asentamiento "Diosece do Alto Solimues").

Alianzas público-privadas, con la Academia y centros de investigación junto con entidades vivas del territorio y actores privados interesados, con el fin de crear una red de pensamiento y acción concreta que vigile y promulgue un desarrollo sostenible del territorio.

Promover la participación de universidades públicas y privadas de ambas naciones en trabajo participativo con la comunidad y en el territorio específico, con fines de integrar a la comunidad en una sana apropiación del lugar y de una adecuada construcción social del territorio bajo parámetros de sostenibilidad y cooperación constructiva.

\section{Franja específica de estudio}

La franja de territorio limítrofe transnacional de las ciudades de Leticia en Colombia y Tabatinga en el Brasil comprende los barrios La Unión y Castañal, Colombia, Jorge Eliécer Gaitán, Iane y Ciudad 


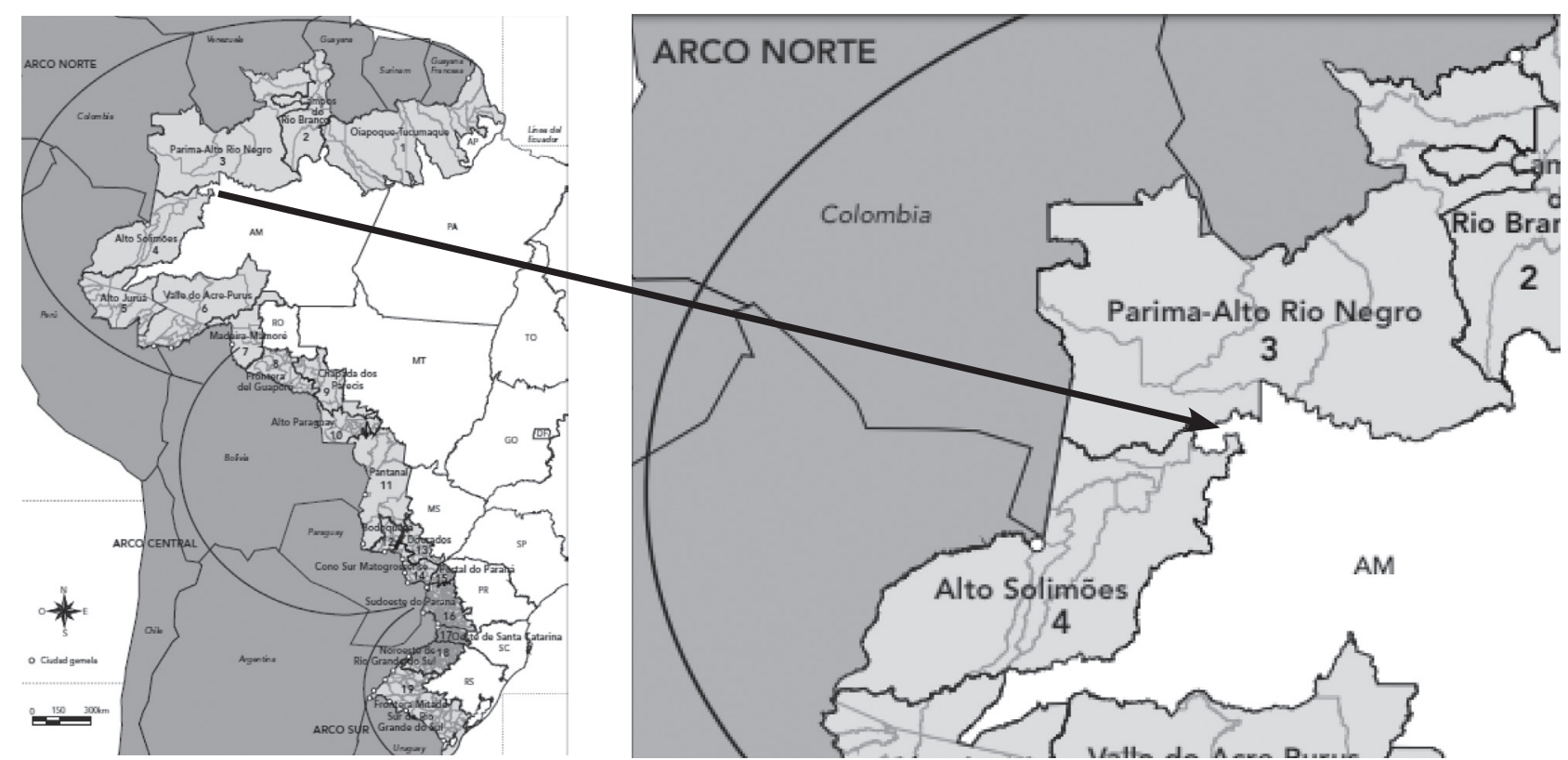

Figura 4. Franja de Planificación Alto Solimues. Estudio de caso: Condiciones de salud, marco geográfico. Fuente: elaboración propia

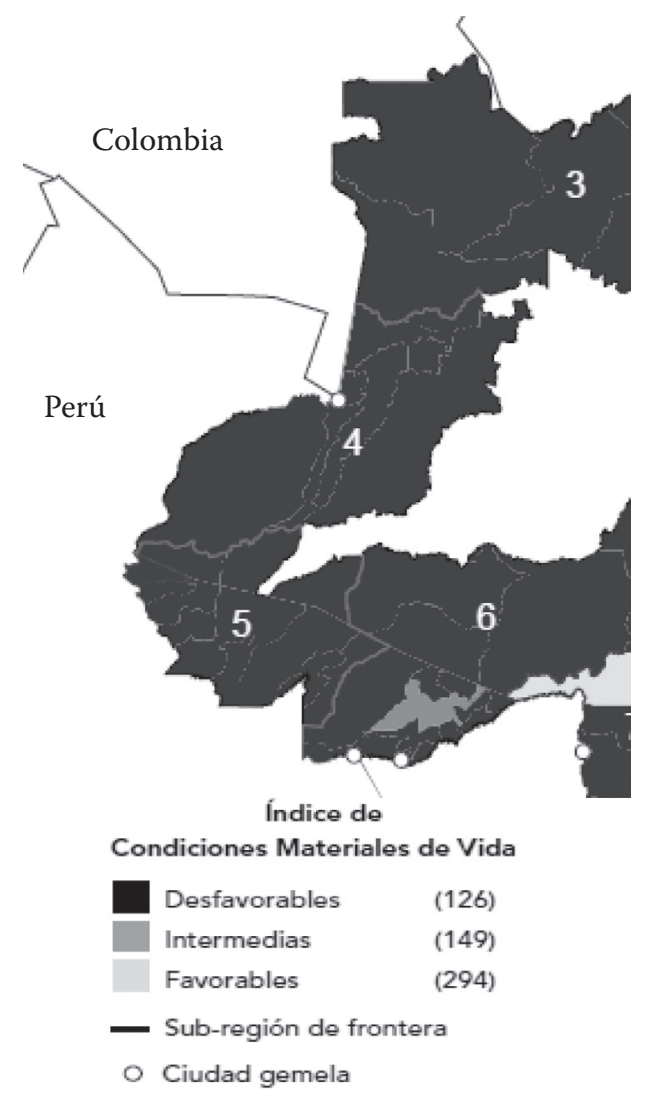

Figura 5. Franja de Planificación Alto Solimues. Estudio de caso: Condiciones de salud, marco geográfico. Fuente: Ministerio de Integración Regional; Grupo Retis/Universidad Federal de Río de Janeiro, 2005
Nueva en Leticia; Guadalupe, São Francisco y el asentamiento "Diosece do Alto Solimues" y los asentamientos del costado nor oriental en el Brasil. Esto en un contexto urbano con características particulares, en tópicos tales como estructuras físicas, saneamiento básico, vulnerabilidad a factores de riesgo ambiental, de remoción en masa y de inundación, tala indiscriminada del sistema biótico, apropiación espacio territorial, tendencias de crecimiento territorial, en la quebrada San Antonio y hacia el río Amazonas.

Es de destacar que en su proceso de expansión territorial tanto en Leticia como en Tabatinga se denota que las estructuras espaciales han sido el resultado de la técnica generada por la sociedad en la geografía, por la concentración variada de fuerzas económicas y culturales, por los lugares de habitación de poblaciones indígenas que se manifiestan en hábitats tipo palafítico con estacones, tarimas y cerramientos en madera local; mientras que los moradores de mayor capacidad económica y de poder habitan en estructuras de material pétreo y con condiciones de infraestructura de vías y caminos en material cementante. En todo caso ambos componentes acusan condiciones 


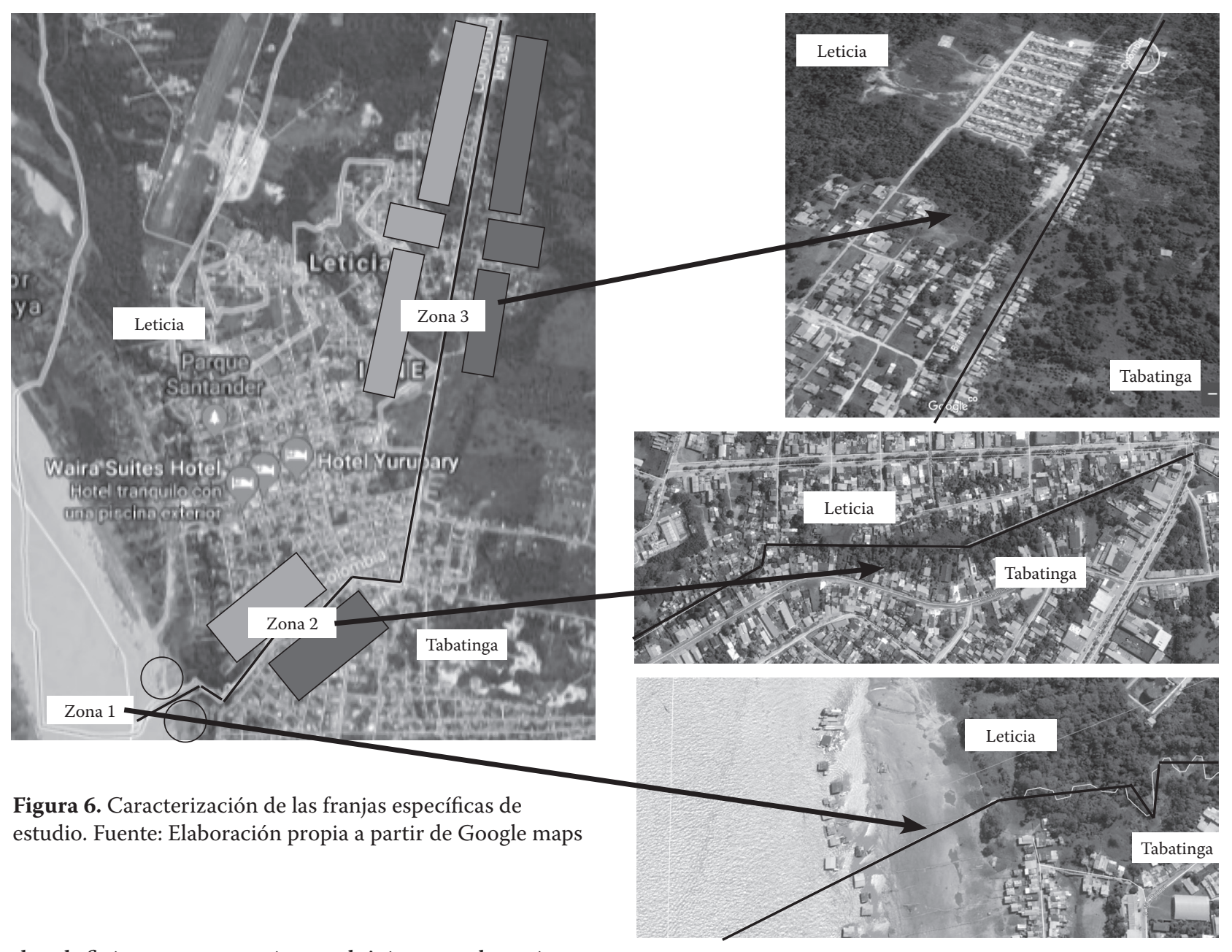

de deficiente saneamiento básico y alto riesgo ambiental que en el caso de la colindancia con la Quebrada San Antonio, tributan residuos contaminantes a la misma y por ende al río Amazonas, que está obrando como fuente de abastecimiento a los Acueductos de ambas ciudades, lo cual hace un urgente el tratamiento adecuado de las aguas residuales.

Se identifican claramente las siguientes sub-franjas de frontera:

- Zona 1: barrios la Unión y comunidad indígena Yagua (Colombia) y del barrio Guadalupe y habitats sobre la Rua Marechal Rondon en Tabatinga (Brasil). Observación: Arquitectura palafítica en madera. Accesibilidad precaria. Deficiente saneamiento básico. Alto riesgo ambiental por inundación, vectores generadores de enfermedades tropicales e IRA.

- Zona 2: polígono de influencia transnacional del Barrio castañal que linda con la calle $3^{a}$ por el norte y por el sur con la quebrada San Antonio y con habitats sobre la Rua Marechal Rondon en Tabatinga (Brasil); hasta la confluencia en el nodo limítrofe internacional dado en la avenida internacional en Leticia y en los inmuebles localizados sobre la Rua de la Amizade en Tabatinga. Observaciones: mediano riesgo ambiental. Alto nivel de contaminación de cuerpos hídricos.

- Materialidad constructiva deficiente. Accesibilidad deficiente a los hábitats en la zona de borde de quebrada y línea fronteriza, costado colombiano. 

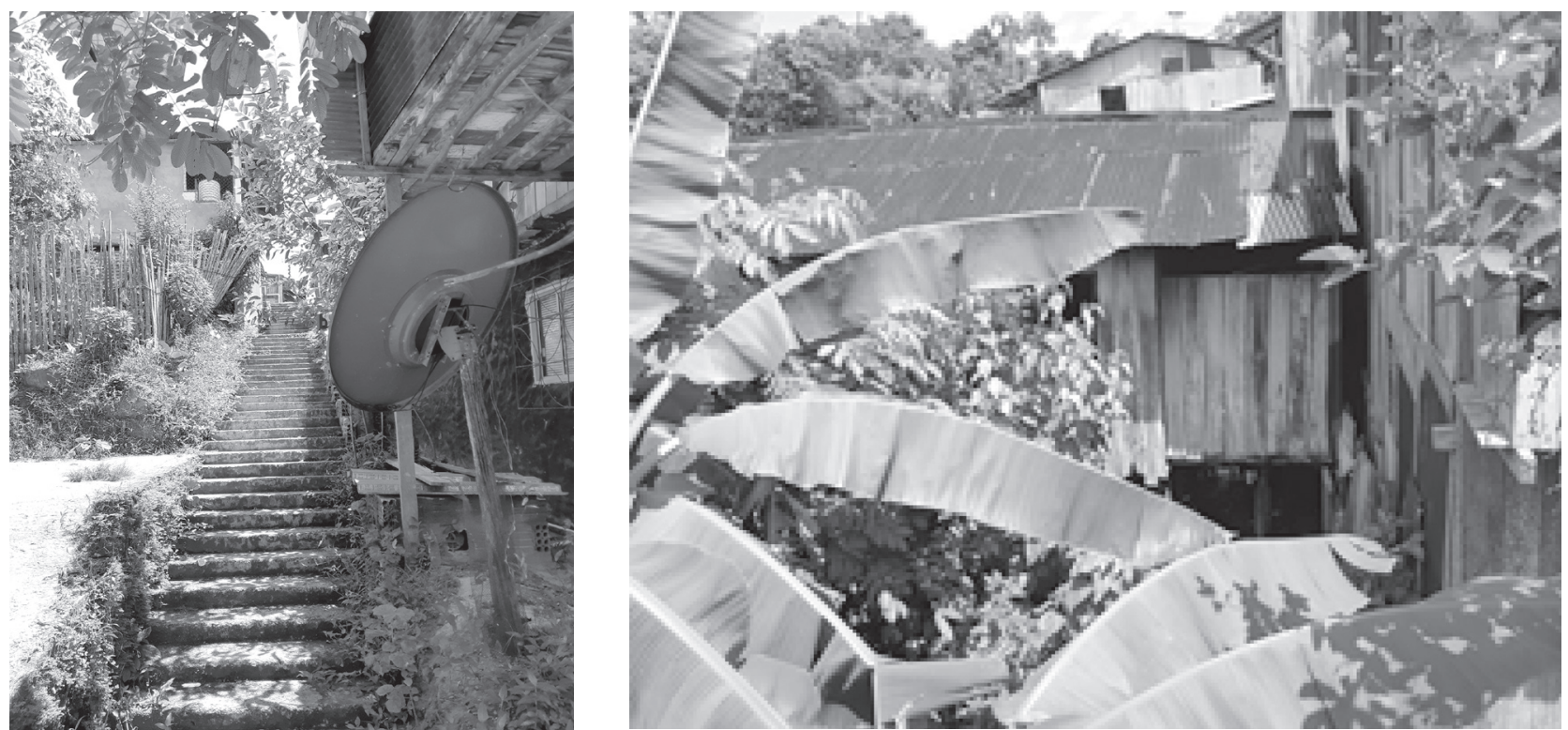

Figuras 7 y 8. Tipologías en frontera costado colombiano, Quebrada San Antonio. Fuente: elaboración propia
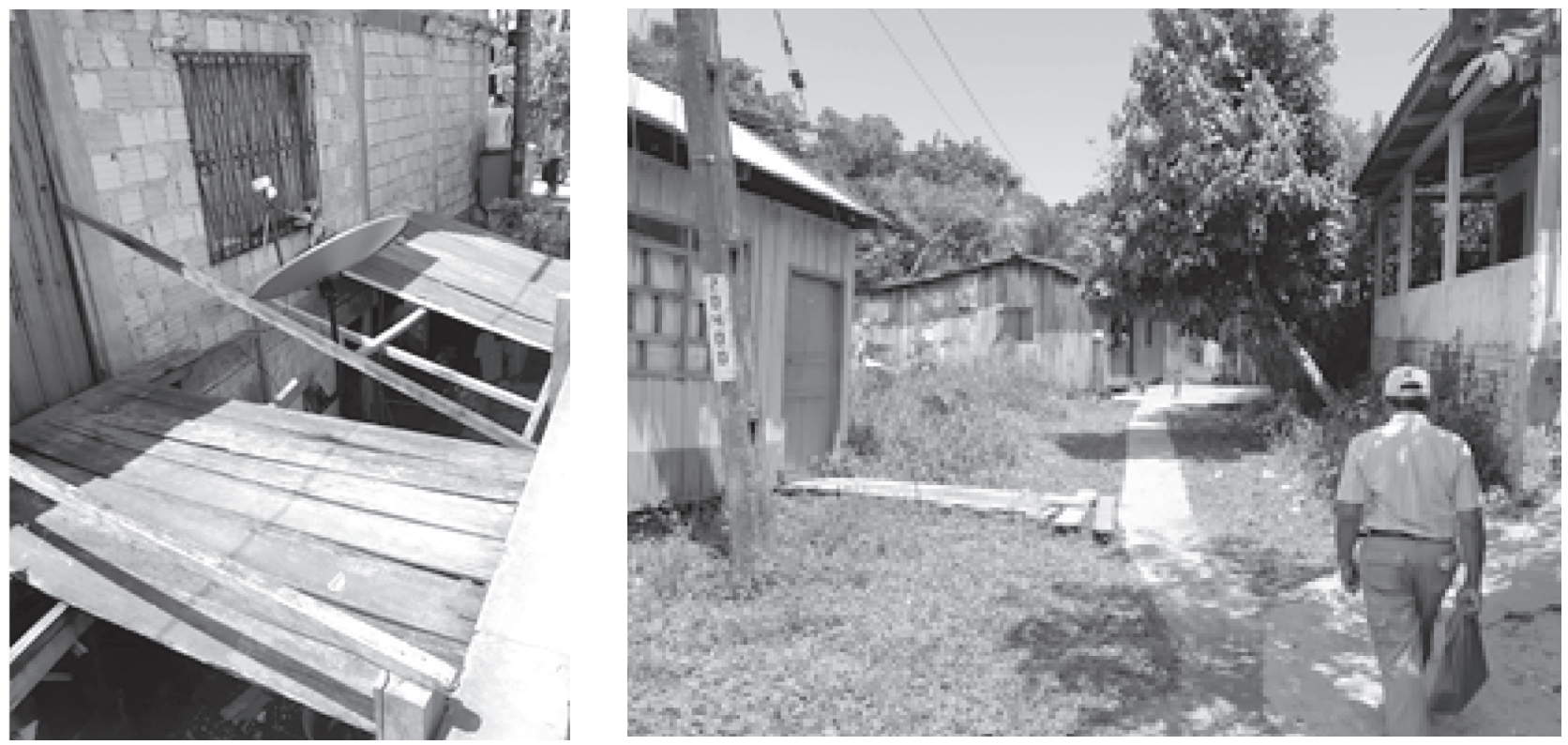

Figuras 9 y 10. Flujos y recorridos en frontera costado colombiano Quebrada San Antonio. Fuente: elaboración propia

Materialidad constructiva y accesibilidad aceptable en los hábitats aledaños a la carrera $3^{\text {a }}$ en Leticia. Se observa urbanismo aceptable en cuanto a vías y andenes sobre el costado de Tabatiga, Rua Marechal Rondon. No presenta servicio básico de alcantarillado, ya que los predios tributan por su parte posterior las aguas servidas, directamente a la quebrada San Antonio.
Zona 3: polígono de influencia transnacional de los barrios Iane y Ciudad Nueva sumado a algunos hábitats de desarrollo espontáneo adheridos a los bordes de los mismos. Esto en relación fronteriza con los asentamientos brasileños que presentan laxitud de control ciudadano y estatal a procesos de deforestación y ampliación de lo construido sin un orden sostenible. 

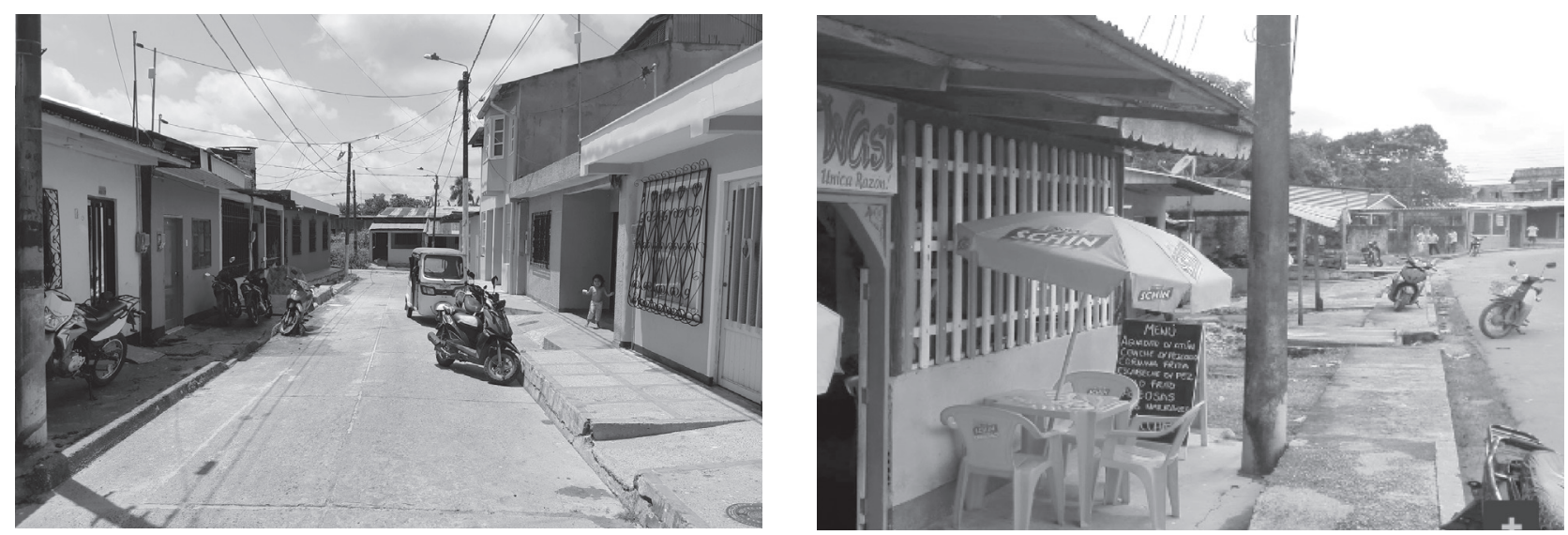

Figuras 11 y 12. Materialidad constructiva y accesibilidad aceptable en los hábitats aledaños a la carrera $3^{\text {a }}$ en Leticia. Fuente: elaboración propia

Se presentan allí modelos específicos de convivencia territorial en su cotidianidad, con lazos comerciales, familiares, laborales y de intercambio en general, desde el Barrio Castañal, que linda con la calle $3^{a}$ por el norte y por el sur con la quebrada San Antonio y con habitates sobre la Rua Marechal Rondon en Tabatinga (Brasil), hasta la confluencia en el nodo limítrofe internacional dado en la avenida internacional en Leticia y en los inmuebles localizados sobre la Rua de la Amizade en Tabatinga.

Se evidencia la similitud de las difíciles condiciones de vida de los habitantes en los dos países,

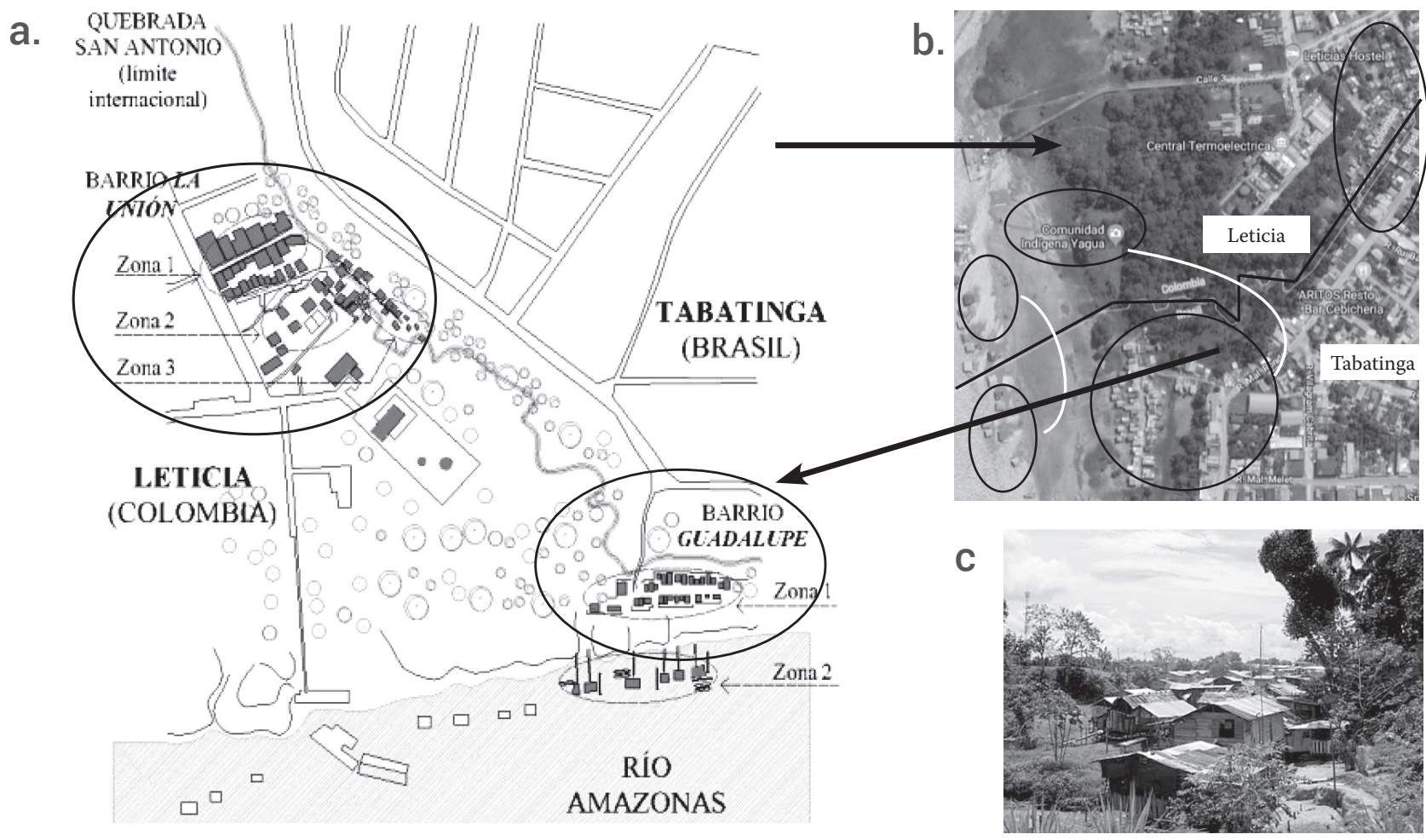

Figura 13. Fuente: a. Elaboraación propia a partir de Google maps y Universidad Nacional. b. Google maps. c. Erik Vergel Tovar. Barrio Guadalupe (Tabatinga) Viviendas flotantes y Palafíticas 

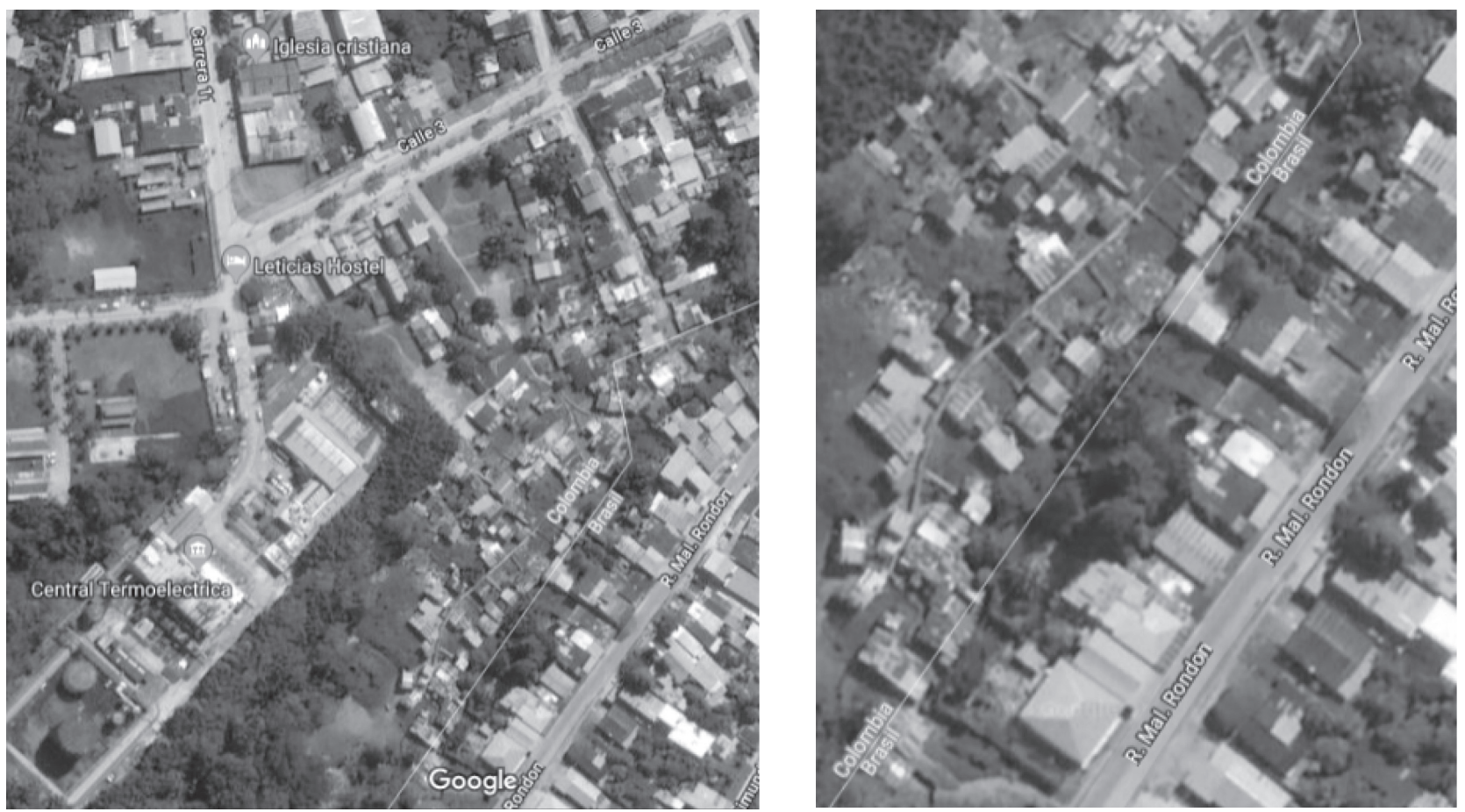

Figura 14. Zonas 1, 2 , 3 y 4 del barrio La Unión. Detalle Urbano Barrio la Unión en Colombia y detalle Borde Quebrada San Antonio con el Brasil. Fuente: elaboración propia a partir de Google maps

presentándose en el costado inferior de la franja de frontera construcción social del territorio y tipologías de asentamiento flotante y palafítico idénticos, entre la zona 2 del barrio Guadalupe en Brasil y zona ídem de la comunidad indígena Yagua en Colombia, manifestando una identidad dicotómica territorial y de asentamientos. Lo anterior se evidencia en las estructuras de madera con soportes tipo estacón o palafitos y recorridos en puentes de tabla de achapo y quinilla entre otros maderables. O sea que la mixtura entre países que rompe los límites de nacionalidad en razón a la convivencia y construcción del lugar tipológico, se dio previo a la protocolización de las líneas limítrofes, tratados y demás acciones institucionales en el tiempo lugar.

Se evidencia un marcado contraste en el borde limítrofe transnacional entre el barrio La Unión (Colombia), con los asentamientos formales que van sobre la Rua Marechal Rondon en Tabatinga (Brasil). Estando los mismos separados por el afluente natural quebrada San Antonio. E igualmente, tanto los asentamientos informales colombianos como los de material petreo y aparente formalidad de los brasileiros, tributan aguas servidas sin tratamiento adecuado y desechos a dicha quebrada. Igualmente se evidencia amenaza por inundación en los asentamientos aledaños a la quebrada San Antonio.

\section{Conclusiones}

Atendiendo entonces a lo dispuesto en el Marco de Acción de Hyogo, se busca ir en concordancia con sus metas para 2005-2015 (aunque ya nos hallemos en 2018 es vigente y proyectable), dentro de las cuales está aumentar la resiliencia de las naciones y las comunidades ante los eventos contra el ecosistema, haciendo énfasis en el papel que deben cumplir los gobiernos y los actores locales, así como en la importancia de involucrar y movilizar a los ciudadanos y comunidades. Es importante indicar que para nuestra franja fronteriza en estudio, Leticia - Tabatinga, los riesgos son de tipo ambiental y a ello se suman los estresores 
en el hábitat y en la salud, que debe ser enfocada en espacios saludables tanto a escala domiciliar como peri domiciliar.

Se identifican diversas vulnerabilidades ecosistémicas, ambientales, bioclimáticas, de inundación y demás factores de habitabilidad, en dicha franja fronteriza, con carácter dinámico y adaptativo, para lo cual es menester definir estrategias de manejo sostenible y recomendaciones de acción en desarrollo territorial, geopolítica y normatividad.

Tabatinga ejerce una función nódulo de la red transnacional debido al desarrollo histórico que constituyó dicho asentamiento en la regón trifronteriza y con un alto contenido de ocupación estratégica militar con criterioos de habitabilidad y territorialidad otorgado por las familias, prestadores de servicios y redes de apoyo a dichas acciones de estrategia estatal desde el concepto de preservación de la frontera.

Es determinante manifestar que no hay fronteras en el bioma ecosistémico y que éste debe generar una red de interconexión de elementos que lo protejan y lo hagan autosustentable. De ahí la importancia de recomendar que toda acción y gestión de intervención o estudio del lugar tenga en cuenta esta convivencia entre la unidad ecosistémica y las divisiones fronterizas espaciales y legales.

En lo que respecta al componente de la sustentabilidad urbana, se retomaron algunos elementos teóricos relacionados con los criterios de evaluación del Programa 21 (Barton, 2006), ya que varios de éstos aplican tanto a los parámetros de resiliencia, como a la sustentabilidad, ambos en lo urbano. Allí, el gran tema de la sustentabilidad urbana como planificación estratégica se condensa en tópicos concernientes a la franja de estudio, tales como el enfrentar las necesidades económicas, sociales y ecológicas de estos habitantes, en forma conjunta. En tal sentido también debe haber un consenso participativo hacia la definición de una visión de un futuro sustentable y prepararse un plan de acción para acercarse a resultados en cuanto a sustentabilidad urbana e implementarse un sistema económico que pueda generar superávit y conocimiento técnico en una forma autosuficiente y sostenida. Un sistema de producción que respete la obligación de preservar la base ecológica del desarrollo y finalmente un sistema administrativo que sea flexible y tenga la capacidad de autocorrección.

\section{Referencias bibliográficas}

Asociación Zonal de Consejo de Autoridades indígenas de Tradición autóctono AZCAITA. (2008). Simposio: Plan de Vida de los Pueblos Tikuna, Uitoto, Cocama y Yagua. Leticia, Amazonas, Colombia.

Barton R., J. (2006). "Sustentabilidad urbana como planificación Estratégica”. Revista Eure. Vol. XXXII, No 96, pp. 27-45.

Lucca, E. (2016). La gestión de los territorios: la cosecha escondida o la percepción ambiental de los espacios. Recuperado de https://ebookcentral.proquest.com

Peiter, P. C. (2007). Condiciones de vida, situación de la salud y disponibilidad de servicios de salud en la frontera de Brasil: un enfoque geográfico. Scielo. pp. 237-250.

Picón A., Jorge E. (2010). “Transformación Urbana de Leticia. Énfasis en el Período 1950-1960. La Construcción de una ciudad en la selva amazónica y en la región trifronteriza" Bogotá, Colombia-: Editorial gente Nueva.

Riaño, E. y Salazar, C. A. (2009). Sistema urbano en la Región Amazónica colombiana. Leticia, Amazonas: Sinchi.

Schor, T. (2013). As cidades invisíveis da Amazônia brasileira. Mercator-Revista de Geografía da UFC, 12(28), pp. 67-84.

Teixeira da Silva, A. (2009). Amazonia: Geopolítica, Integración y Seguridad Multidimensional. Temas del cono sur: Dossier de integración 
número 57. Recuperado de https://ebookcentral.proquest.com

Tratado de Cooperación Amazónica. Recuperado de http://otca.info/portal/admin/_upload/trata do/TRATADO_COOPERACION_AMAZONICA_ESP.pdf

Zarate, C. (2008). “Silvícolas, siringueros y agentes estatales. El surgimiento de una sociedad transfronteriza en la Amazonía de Brasil, Perú y
Colombia 1880-1932". Mundo Amazónico, 1, pp. 349-354. Citado por Cabrera, G. (2010).

Zarate, C. (2012). Ciudades pares en la frontera amazónica colonial y republicana. En Zarate, C., Espacios urbanos y sociedades transfronterizas en la Amazonía, pp. 21-44. Leticia, Amazonas, Colombia: Universidad Nacional de Colombia, Sede Amazonia. Instituto Amazónico de investigaciones. Imani. 